\title{
Algebraic Methods for Counting Euclidean Embeddings of Rigid Graphs
}

\author{
Ioannis Z. Emiris ${ }^{1}$, Elias P. Tsigaridas ${ }^{2}$, and Antonios E. Varvitsiotis ${ }^{3}$ \\ 1 University of Athens, Athens, Greece \\ 2 INRIA Méditerranée, Sophia-Antipolis, France \\ 3 CWI, Amsterdam, The Netherlands
}

\begin{abstract}
The study of (minimally) rigid graphs is motivated by numerous applications, mostly in robotics and bioinformatics. A major open problem concerns the number of embeddings of such graphs, up to rigid motions, in Euclidean space. We capture embeddability by polynomial systems with suitable structure, so that their mixed volume, which bounds the number of common roots, to yield interesting upper bounds on the number of embeddings. We focus on $\mathbb{R}^{2}$ and $\mathbb{R}^{3}$, where Laman graphs and 1-skeleta of convex simplicial polyhedra, respectively, admit inductive Henneberg constructions. We establish the first general lower bound in $\mathbb{R}^{3}$ of about $2.52^{n}$, where $n$ denotes the number of vertices. Moreover, our implementation yields upper bounds for $n \leq 10$ in $\mathbb{R}^{2}$ and $\mathbb{R}^{3}$, which reduce the existing gaps, and tight bounds up to $n=7$ in $\mathbb{R}^{3}$.
\end{abstract}

Keywords: Rigid graph, Euclidean embedding, Henneberg construction, polynomial system, root bound, cyclohexane caterpillar.

\section{Introduction}

Rigid graphs (or frameworks) constitute an old but still active area of research due to certain deep mathematical questions, as well as numerous applications, e.g. mechanism theory [89, and structural bioinformatics 4].

Given graph $G=(V, E)$ and a collection of edge lengths $l_{i j} \in \mathbb{R}_{>0}$, for $(i, j) \in$ $E$, a Euclidean embedding in $\mathbb{R}^{d}, d \geq 1$ is a mapping of the vertices $V$ to points in $\mathbb{R}^{d}$, such that $l_{i j}$ equals the Euclidean distance between the images of the $i$-th and $j$-th vertices, for all edges $(i, j) \in E$. There is no requirement on whether the edges cross or not. $G$ is generically rigid in $\mathbb{R}^{d}$ if, for generic edge lengths, it admits a finite number of embeddings in $\mathbb{R}^{d}$, modulo rigid motions. $G$ is minimally rigid if it is no longer rigid once any edge is removed. In the sequel, generically minimally rigid graphs are referred to as rigid.

A graph is called Laman if $|E|=2|V|-3$ and, additionally, all of its induced subgraphs on $k<|V|$ vertices have $\leq 2 k-3$ edges. The Laman graphs are precisely the rigid graphs in $\mathbb{R}^{2}$; they also admit inductive constructions. In $\mathbb{R}^{3}$ there is no analogous combinatorial characterization of rigid graphs, but the 1-skeleta, or edge graphs, of (convex) simplicial polyhedra are rigid in $\mathbb{R}^{3}$, and admit inductive constructions. 


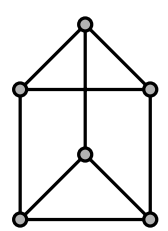

Fig. 1. The Desargues graph

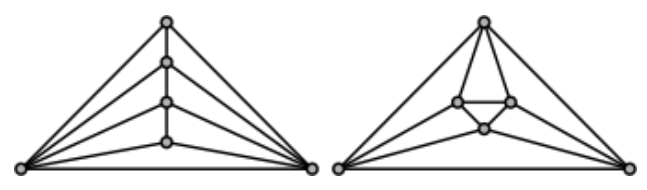

Fig. 2. All simplicial polyhedra for $n=6$

We deal with the problem of computing the maximum number of distinct planar and spatial Euclidean embeddings of rigid graphs, up to rigid motions, as a function of the number of vertices. To study upper bounds, we define a square polynomial system, expressing the edge length constraints, whose real solutions correspond precisely to the different embeddings. Here is a system expressing embeddability in $\mathbb{R}^{3}$, where $\left(x_{i}, y_{i}, z_{i}\right)$ are the coordinates of the $i$-th vertex, and 3 vertices are fixed to discard translations and rotations:

$$
\begin{cases}x_{i}=a_{i}, y_{i}=b_{i}, z_{i}=c_{i}, & i=1,2,3, \quad a_{i}, b_{i}, c_{i} \in \mathbb{R}, \\ \left(x_{i}-x_{j}\right)^{2}+\left(y_{i}-y_{j}\right)^{2}+\left(z_{i}-z_{j}\right)^{2}=l_{i j}^{2}, & (i, j) \in E-\{(1,2),(1,3),(2,3)\}\end{cases}
$$

All nontrivial equations are quadratic; there are $2 n-4$ for Laman graphs, and $3 n-9$ for 1-skeleta of simplicial polyhedra, where $n$ is the number of vertices. Bézout's bound on the number of complex roots equals the product of the degrees, hence $4^{n-2}$ and $8^{n-3}$, respectively.

For the planar and spatial case, the best upper bounds are $\left(\begin{array}{c}2 n-4 \\ n-2\end{array}\right)$ and $\frac{2^{n-3}}{n-2}\left(\begin{array}{c}2 n-6 \\ n-3\end{array}\right)$ 2. In applications, it is crucial to know the number of embeddings for small $n$. The main result in this direction was to show that the Desargues (or 3 -prism) graph (Figure 1) admits 24 embeddings in $\mathbb{R}^{2}$ [2. This led the same authors to lower bounds in $\mathbb{R}^{2}: 24^{\lfloor(n-2) / 4\rfloor} \simeq 2.21^{n}$ by a "caterpillar" constructed by concatenating copies of the Desargues graph, and $2 \cdot 12^{\lfloor(n-3) / 3\rfloor} \simeq 2.29^{n} / 6$ obtained by a Desargues "fan" 1 .

Bernstein's bound on the number of roots of a polynomial system exploits the sparseness of the equations. It is bounded by Bézout's bound and typically much tighter. We have developed software to construct all rigid graphs up to isomorphism, for small $n$, and compute the Bernstein's bounds. Besides some straightforward upper bounds in Lemmas 1 and 6. our main contribution is twofold. We derive the first general lower bound in $\mathbb{R}^{3}$ :

$$
16^{\lfloor(n-3) / 3\rfloor} \simeq 2.52^{n}, \quad n \geq 9,
$$

by designing a cyclohexane caterpillar. We also obtain improved upper and lower bounds for $n \leq 10$ in $\mathbb{R}^{2}$ and $\mathbb{R}^{3}$ (Tables 1 and 2). Moreover, we establish tight bounds for $n \leq 7$ in $\mathbb{R}^{3}$ by appropriately formulating the polynomial system. We apply Bernstein's Second theorem to show that the above polynomial system cannot yield tight bounds.

\footnotetext{
${ }^{1}$ We have corrected the exponent of the original statement.
} 
The rest of the paper is structured as follows: Section 2 discusses the planar case, Section 3 presents our algebraic tools, Section 4 deals with $\mathbb{R}^{3}$, and we conclude with open questions.

Some results appeared in [5] in preliminary form.

\section{Planar Embeddings of Laman Graphs}

Laman graphs admit inductive constructions, starting with a triangle, and followed by a sequence of Henneberg-1 (or $H_{1}$ ) and Henneberg-2 steps (or $H_{2}$ ). Each step adds a new vertex: $H_{1}$ connects it to two existing vertices, $H_{2}$ connects it to 3 existing vertices having at least one edge among them, which is removed. We represent a Laman graph by its Henneberg sequence $\triangle s_{4} \ldots, s_{n}$, where $s_{i} \in\{1,2\}$. A Laman graph is called $H_{1}$ if and only if it can be constructed using only $H_{1}$; otherwise it is called $H_{2}$. Since two generic circles intersect in two real points, $H_{1}$ exactly doubles the maximum number of embeddings. It follows that a $H_{1}$ graph has $2^{n-2}$ embeddings.

One can easily verify that every $\triangle 2$ graph is isomorphic to a $\triangle 1$ graph and that every $\triangle 12$ graph is isomorphic to a $\triangle 11$ graph. Consequently, all Laman graphs with $n=4,5$ are $H_{1}$, with 4 and 8 embeddings, respectively. For $n=6$, there are 3 possibilities: the graph is either $H_{1}, K_{3,3}$, or the Desargues graph. Since the $K_{3,3}$ graph has at most 16 embeddings [89] and the Desargues graph has 24 embeddings [2], the upper bound is 24 .

Using our software (Section [3), we construct all Laman graphs for $n=$ $7, \ldots, 10$, compute the respective Bernstein's bounds, and bound the maximum number of Euclidean embeddings by 64, 128, 512 and 2048, respectively. Table 1 summarizes our results for $n \leq 10$. The lower bound for $n=9$ follows from the Desargues fan. All other lower bounds follow from the fact that $H_{1}$ doubles the number of embeddings.

We now establish a "sparse" upper bound.

Lemma 1. Let $G$ be Laman, with $k \geq 4$ degree-2 vertices. Then, the number of its planar embeddings is at most $2^{k-4} 4^{n-k}$.

Proof. There are at least $k$ removals of degree- 2 vertices, since all created graphs are Laman. The final graph has a Bézout bound of $4^{n-k}$.

\section{An Algebraic Interlude}

Given a polynomial $f$ in $n$ variables, its support is the set of exponents in $\mathbb{N}^{n}$ corresponding to nonzero terms (or monomials). The Newton polytope of $f$ is the convex hull of its support and lies in $\mathbb{R}^{n}$. Consider polytopes $P_{i} \subset \mathbb{R}^{n}$ and $\lambda_{i} \in \mathbb{R}, \lambda_{i} \geq 0$, for $i=1, \ldots, n$. Consider the Minkowski sum $\lambda_{1} P_{1}+\cdots+\lambda_{n} P_{n} \in$ $\mathbb{R}^{n}$ : its (Euclidean) volume is a homogeneous polynomial of degree $n$ in the $\lambda_{i}$. The coefficient of $\lambda_{1} \cdots \lambda_{n}$ is the mixed volume of $P_{1}, \ldots, P_{n}$. If $P_{1}=\cdots=P_{n}$, then the mixed volume is $n$ ! times the volume of $P_{1}$. We focus on $\mathbb{C}^{*}=\mathbb{C}-\{0\}$. 
Theorem 2. 1] Let $f_{1}=\cdots=f_{n}=0$ be a polynomial system in $n$ variables with real coefficients, where the $f_{i}$ have fixed supports. The number of isolated common solutions in $\left(\mathbb{C}^{*}\right)^{n}$ is bounded above by the mixed volume of (the Newton polytopes of) the $f_{i}$. This bound is tight for generic coefficients of the $f_{i}$ 's.

Bernstein's Second theorem below was used in $\mathbb{R}^{2}\left[7\right.$; we apply it to $\mathbb{R}^{3}$. Given $v \in \mathbb{R}^{n}-\{0\}$ and polynomial $f_{i}, \partial_{v} f_{i}$ is the polynomial obtained by keeping only the terms whose exponents minimize inner product with $v$; its Newton polytope is the face of the Newton polytope of $f_{i}$ supported by $v$.

Theorem 3. [1] If for all $v \in \mathbb{R}^{n}-\{0\}$ the face system $\partial_{v} f_{1}=\ldots=\partial_{v} f_{n}=0$ has no solutions in $\left(\mathbb{C}^{*}\right)^{n}$, then the mixed volume of the $f_{i}$ exactly equals the number of solutions in $\left(\mathbb{C}^{*}\right)^{n}$, and all solutions are isolated. Otherwise, the mixed volume is a strict upper bound.

In order to bound the number of embeddings of rigid graphs, we have developed specialized software that constructs all Laman graphs and all 1-skeleta of simplicial polyhedra with $n \leq 10$. Our computational platform is SAGE2 2 . We construct all graphs using the Henneberg steps, which we implemented in Python, using SAGE's interpreter. We classify all graphs up to isomorphism using SAGE's interface with N.I.C.E., an open-source isomorphism check software, keeping, for each graph, the Henneberg sequence with the fewest $H_{1}$ steps.

For each graph we construct a system whose real solutions express all embeddings, by formulation (2). We bound the number of its (complex) solutions by mixed volume. For every Laman graph, to discard translations and rotations, we fix one edge to be of unit length, aligned with an axis, with one vertex at the origin. In $\mathbb{R}^{3}$, a third vertex is fixed in a coordinate plane. Depending on the choice of the fixed edge, we obtain different systems hence different mixed volumes, and we keep their minimum.

We used an Intel Core2, at 2.4GHz, with 2GB of RAM. We tested more that 20, 000 graphs, computed the mixed volume of more than 40, 000 systems, taking a total time of about 2 days. Tables 1 and 2 summarize our results.

\section{Spatial Embeddings of 1-Skeleta of Simplicial Polyhedra}

Let us examine 1-skeleta of (convex) simplicial polyhedra, which are rigid in $\mathbb{R}^{3}$ 6]. For such a graph $(V, E),|E|=3|V|-6$ and all induced subgraphs on $k<|V|$ vertices have $\leq 3 k-6$ edges. Consider any $k+2$ vertices forming a cycle with $\geq k-1$ diagonals, $k \geq 1$. The extended Henneberg- $k$ step (or $H_{k}$ ), $k=1,2,3$, corresponds to adding a vertex, connecting it to the $k+2$ vertices, and removing $k-1$ diagonals among them. A graph is the 1-skeleton of a simplicial polyhedron in $\mathbb{R}^{3}$ if and only if it has a construction starting with the 3 -simplex, followed by any sequence of $H_{1}, H_{2}, H_{3}$ [3].

${ }^{2}$ http://www.sagemath.org/ 
Since 3 spheres intersect generically in two points, $H_{1}$ exactly doubles the maximum number of embeddings. In order to discard translations and rotations, we fix a (triangular) facet of the polytope; we choose, without loss of generality, the first 3 vertices and obtain system (1) of dimension $3 n$. Let $v=$ $(0,0,0,0,0,0,0,0,0,-1, \ldots,-1) \in \mathbb{R}^{3 n}$, the face system is:

$$
\begin{cases}x_{i}=a_{i}, y_{i}=b_{i}, z_{i}=c_{i}, & i=1,2,3, \quad a_{i}, b_{i}, c_{i} \in \mathbb{R}, \\ \left(x_{i}-x_{j}\right)^{2}+\left(y_{i}-y_{j}\right)^{2}+\left(z_{i}-z_{j}\right)^{2}=0, & (i, j) \in E, i, j \notin\{1,2,3\}, \\ x_{i}^{2}+y_{i}^{2}+z_{i}^{2}=0, & (i, j) \in E: i \notin\{1,2,3\}, j \in\{1,2,3\} .\end{cases}
$$

This system has $\left(a_{1}, b_{1}, c_{1}, \ldots, a_{3}, b_{3}, c_{3}, 1,1, \gamma \sqrt{2}, \ldots, 1,1, \gamma \sqrt{2}\right) \in\left(\mathbb{C}^{*}\right)^{3 n}$ as a solution, where $\gamma= \pm \sqrt{-1}$. According to Theorem 3 , the mixed volume is not a tight bound on the number of solutions in $\left(\mathbb{C}^{*}\right)^{3 n}$. This was also observed, for $\mathbb{R}^{2}$, in [7]. To remove spurious solutions let $w_{i}=x_{i}^{2}+y_{i}^{2}+z_{i}^{2}$, for $i=1, \ldots, n$. This yields an equivalent system, with lower mixed volume, which will be used in our computations:

$$
\begin{cases}x_{i}=a_{i}, y_{i}=b_{i}, z_{i}=c_{i}, & i=1,2,3 \\ w_{i}=x_{i}^{2}+y_{i}^{2}+z_{i}^{2}, & i=1, \ldots, n, \\ w_{i}+w_{j}-2 x_{i} x_{j}-2 y_{i} y_{j}-2 z_{i} z_{j}=l_{i j}^{2}, & (i, j) \in E-\{(1,2),(1,3),(2,3)\} .\end{cases}
$$

For $n=4$, the only simplicial polytope is the 3-simplex, which has 2 embeddings. For $n=5$, there is a unique 1-skeleton of a simplicial polyhedron [3, and is obtained from the 3 -simplex by $H_{1}$, hence it has exactly 4 embeddings.

For $n=6$, there are two non-isomorphic graphs $G_{1}, G_{2}$ (Figure 2] [3], yielding respective mixed volumes of 8 and $16 . G_{2}$ is the graph of the cyclohexane, which admits 16 different embeddings [4. To see this, the cyclohexane is a 6-cycle with known lengths between vertices at distance 1 (adjacent) and 2 . Alternatively, $G_{2}$ corresponds to a Stewart platform parallel robot with 16 configurations, where triangles define the platform and base, and 6 lengths link the triangles in a jigsaw shape. This proves:

Lemma 4. The 1-skeleton of a simplicial polyhedron with $n=6$ has at most 16 embeddings and this is tight.

Let us glue copies of cyclohexanes sharing a triangle, each adding 3 vertices, thus obtaining the 1-skeleton of a simplicial polytope. Applying Lemma 4 we have:

Theorem 5. There exist edge lengths for which the cyclohexane caterpillar construction has $16^{\lfloor(n-3) / 3\rfloor} \simeq 2.52^{n}$ embeddings, for $n \geq 9$.

Table 2 summarizes our results for $n \leq 10$. The upper bounds for $n=7, \ldots, 10$ are computed by our software. The lower bound for $n=9$ is from Theorem 5 . All other lower bounds are obtained by considering a $H_{1}$ construction.

We state without proof a result similar to Lemma 1.

Lemma 6. Let $G$ be the 1-skeleton of a simplicial polyhedron with $k \geq 9$ degree3 vertices. The number of embeddings of $G$ is bounded above by $2^{k-9} 8^{n-k}$. 
Table 1. Bounds for Laman graphs

\begin{tabular}{|c||c|c|c|c|c|c|c|c|}
\hline $\mathrm{n}=$ & 3 & 4 & 5 & 6 & 7 & 8 & 9 & 10 \\
\hline \hline lower & 2 & 4 & 8 & 24 & 48 & 96 & 288 & 576 \\
\hline upper & 2 & 4 & 8 & 24 & 64 & 128 & 512 & 2048 \\
\hline
\end{tabular}

Table 2. Bounds for 1-skeleta of simplicial polyhedra

\begin{tabular}{|c||c|c|c|c|c|c|c|}
\hline $\mathrm{n}=$ & 4 & 5 & 6 & 7 & 8 & 9 & 10 \\
\hline \hline lower & 2 & 4 & 16 & 32 & 64 & 256 & 512 \\
\hline \hline upper & 2 & 4 & 16 & 32 & 160 & 640 & 2560 \\
\hline
\end{tabular}

\section{Further Work}

The most important problem in rigidity theory is the combinatorial characterization of rigid graphs in $\mathbb{R}^{3}$. Since we rely on Henneberg constructions, it is crucial to determine the effect of each step on the number of embeddings: we conjecture that $H_{2}$ multiplies it by $\leq 4$ and $H_{3}$ by $\leq 8$, but these may not always be tight. Our conjecture has been verified for small $n$.

Acknowledgments. A.V. performed this work as part of his Master's thesis at University of Athens; he thanks G. Rote for discussions, and acknowledges partial support by project IST-006413-2 ACS: Algorithms for Complex Shapes. I.E. is partially supported by project PITN-GA-2008-214584 SAGA: Shapes, Geometry, and Algebra; part of this work was carried out while on leave at Ecole Normale de Paris, and Ecole Centrale de Paris. E.T. is partially supported by contract ANR-06-BLAN-0074 "Decotes".

\section{References}

1. Bernstein, D.N.: The number of roots of a system of equations. Fun. Anal. Pril. 9, 1-4 (1975)

2. Borcea, C., Streinu, I.: The number of embeddings of minimally rigid graphs. Discrete Comp. Geometry 31(2), 287-303 (2004)

3. Bowen, R., Fisk, S.: Generation of triangulations of the sphere. Math. of Computation 21(98), 250-252 (1967)

4. Emiris, I.Z., Mourrain, B.: Computer algebra methods for studying and computing molecular conformations. Algorithmica, Special Issue 25, 372-402 (1999)

5. Emiris, I.Z., Varvitsiotis, A.: Counting the number of embeddings of minimally rigid graphs. In: Proc. Europ. Workshop Comput. Geometry, Brussels (2009)

6. Gluck, H.: Almost all simply connected closed surfaces are rigid. Lect. Notes in Math. 438, 225-240 (1975)

7. Steffens, R., Theobald, T.: Mixed volume techniques for embeddings of Laman graphs. In: Proc. Europ. Workshop Comput. Geometry, Nancy, France, pp. 25-28 (2008); Final version accepted in Comp. Geom: Theory \& Appl., Special Issue

8. Walter, D., Husty, M.: On a 9-bar linkage, its possible configurations and conditions for paradoxical mobility. In: IFToMM Congress, Besançon, France (2007)

9. Wunderlich, W.: Gefärlice Annahmen der Trilateration und bewegliche Fachwerke I. Zeitschrift für Angewandte Mathematik und Mechanik 57, 297-304 (1977) 